\title{
ASPECTOS FUNDAMENTAIS DA DETERMINAÇÃO DA EXIGÊNCIA ENERGÉTICA DE CÃES DOMÉSTICOS
}

\author{
Fundamental Aspects of the Determination of Energy \\ Requirement of Domestic Dogs
}

\section{Resumo}

Este trabalho procura mostrar alguns dos principais aspectos que devem ser observados ao alimentar ou elaborar um programa nutricional de cães adultos com base em sua exigência energética. A energia é um dos principais "nutrientes" que devem ser levados em consideração, quando se busca determinar o consumo alimentar e o conseqüente desempenho de um cão, nas mais variadas etapas de sua vida. $\mathrm{O}$ consumo energético é o que, em primeira instância, determinará a quantidade de alimento que será ingerida por um animal. Por isso, torna-se fundamental entender como se faz a determinação da exigência energética para cães.

Palavras-chave: Nutrição; Alimentação; Cães; Exigência energética.

\begin{abstract}
This work search to show some of the fundamental aspects that should be observed when feeding or working out a nutritional program for adult dogs, based on the energy requirement. The energy is one of the main "nutrients" that must be taken into consideration when one searchs to determine the alimentary consumption and the consequent performance of a dog, in the most varied stages of its life. The energy consumption is what in lower court, it will determine the amount of food that will be ingested by an animal. Therefore, it becomes basic to understand how the determination of the energy requirement for dogs works.
\end{abstract}

Keywords: Nutrition; Feeding; Dogs; Energy requirement. 


\section{INTRODUÇÃO}

\section{Descrição da espécie canina}

Os canídeos são mamíferos que se caracterizam por possuir dentes caninos pontiagudos, uma dentição para o regime onívoro e um esqueleto dimensionado para uma locomoção digitígrada. Pertencem à ordem dos carnívoros, cujo desenvolvimento data do início da era terciária, nos locais antes habitados pelos grandes répteis, que desapareceram no final da era secundária (QUADRO 1). Os canídeos do gênero canis surgiram apenas no final do período terciário. Teorias recentes indicam que a espécie Canis familiaris surgiu apenas há 135.000 anos (GRANDJEAN, 2001).

QUADRO 1 - Classificação zoológica do cão

Chart 1 - Dog zoological classification

\begin{tabular}{l|l}
\hline Classe & Mamiferos \\
\hline Subclasse & Eutérios \\
Superordem & Camivoros \\
Ordem & Camivoros terrestres \\
Familia & Canideos \\
Gênero & Canis \\
Espécie & Canis familiaris $(2 \mathrm{n} \rightarrow 78$ cromossomos $)$ \\
\hline
\end{tabular}

Fonte: GRANDJEAN (2001).

Os cães, como os demais carnívoros, estão adaptados a dietas relativamente concentradas e altamente digestíveis e são caracterizados por um intestino simples e curto (AHLSTROM; KENDALL, 1981; SKREDE, 1998).

\section{Comportamento alimentar canino}

Segundo Mohrman (1979), o cão é um animal carnívoro por definição, mas onívoro por convenção, por isso é mais bem definido como sendo um carnívoro não estrito.

A alimentação de cães deve levar em conta que existem grandes diferenças entre os tamanhos dos animais desta espécie (QUADRO 2), onde se encontra desde um Chiuaua com $1 \mathrm{~kg}$ de peso vivo, até um São Bernardo que pode pesar mais de 100 kg (CASE; CAREY; HIRAKAWA, 1998; BURGER; JOHNSON, 1991; EARLE, 1993).

QUADRO 2 - Diferenças fundamentais entre cães de porte mini e gigante Chart 2 - Fundamentals differences between mini and giant dogs

\begin{tabular}{l|l|l|l}
\hline Diferença & Chihuahua & Fator de variação & São Bemardo \\
\hline Crescimento & 8 meses & $3 \mathrm{x}$ maior & 24 mescs \\
Peso adulto médio & $1 \mathrm{~kg}$ & $100 \mathrm{x}$ maior & $100 \mathrm{~kg}$ \\
Amplitude de crescimento & peso nasci, $\mathrm{x} 20$ & $5 \mathrm{x}$ maior & peso nasci, 100 \\
Tamanho dos dentes & canino: $4-5 \mathrm{~mm}$ & $3 \mathrm{x}$ maiores & canino: 5 - $16 \mathrm{~mm}$ \\
Necessidade energética & $132 \mathrm{kcal} / \mathrm{kg} \mathrm{PV}$ & $3 \mathrm{x}$ mais por $\mathrm{kg}$ & $45 \mathrm{kcal} / \mathrm{kg} \mathrm{PV}$ \\
Peso do ap. digestivo & $7 \%$ do PV & mais de $2 \mathrm{x}$ maior & $2,8 \%$ do PV \\
Esperança de vida & $>12$ anos & quase $2 \mathrm{x}$ menor & 7 anos \\
\hline
\end{tabular}

Fonte: GRANDJEAN (2001). 
Além das diferenças características existentes entre as diversas raças, deve-se levar em conta os níveis de atividade física, clima, idade, sexo, composição corporal e outros fatores que possam influenciar a quantidade de alimento ingerido (CASE; CAREY; HIRAKAWA, 1998; CONSTABLE et al., 1996 GRANDJEAN, 2001; NRC, 1985; AAFCO, 1999).

\section{Energia}

Sem levar em consideração a água, a energia é o componente mais importante a se considerar em um alimento, para todo e qualquer animal (CASE; CAREY; HIRAKAWA, 1998).

De acordo com Miller et al. (1965), a unidade de energia usada na nutrição humana e animal é a quilocaloria (kcal), que vem a ser a quantidade de calor (energia) necessária para elevar a temperatura de $1 \mathrm{~g}$ de água em $1^{\circ} \mathrm{C}$. Para converter quilocaloria em quilojoule (kj), unidade utilizada em países europeus, multiplica-se o valor de quilocalorias por 4,18 (CASE; CAREY; HIRAKAWA, 1998).

Segundo o NRC (1985), quando um alimento é completamente oxidado em uma bomba calorimétrica, a energia combustível total liberada é conhecida como energia bruta (EB). A diferença entre a energia bruta (EB) consumida e a energia bruta das fezes é chamada como energia aparentemente digerível (ED). Também ocorrem perdas adicionais de energia na urina e na forma de gases.

Por razões práticas, apenas a energia perdida pela urina é subtraída da ED para determinar a energia metabolizável (EM), já que a quantidade de energia perdida na forma de gases em animais monogástricos é insignificante (GREENHALGH, 1988; MCDONALD; EDWARDS; NRC, 1981).

Burger (1994) determinou em experimentos realizados no Waltham Centre for Pet Nutrition $(W C P N / U K)$ que cerca de $93 \%$ da energia digestível é metabolizada. Tem-se então: EM = 0,93ED (KENDALL; HOLME; SMITH, 1982).

O conteúdo de EM de um alimento é uma expressão válida da quantidade de energia disponível para o cão e uma base para a comparação de vários ingredientes utilizados na alimentação animal. Vale ressaltar que os valores de EM da maioria dos ingredientes utilizados atualmente não foram determinados para cães, por isso estudos adicionais devem ser realizados (NRC, 1985).

Podem-se determinar os valores de EM por meio de fórmulas que calculam um alimento, a partir do conteúdo analisado de carboidratos, gordura e proteína. As fórmulas usadas para analisar os alimentos de cães e gatos incluem constantes que justificam as perdas de energia fecais e urinárias (NRC, 1985).

Segundo o NRC (1985), os valores de EB que representam o conteúdo total de energia para carboidratos (ENN), gordura (EE) e proteína (PB) são de 4,15; 9,40 e 5,65 kcal $/ \mathrm{kg}$, respectivamente. O valor de EB para proteína, quando se corrige a perda de energia do nitrogênio na forma de produtos metabólicos, cai para 4,40 (5,65 - 1,25).

No entanto, os animais são incapazes de aproveitar toda a energia presente nos nutrientes. A pouca eficiência na digestão, absorção e assimilação causam perdas de energia. Nos alimentos para humanos, utilizam-se os fatores de Atwater, de 4,0-9,0-4,0 kcal/g, para calcular os valores de EB para carboidratos, gordura e proteína (CASE; CAREY; HIRAKAWA, 1998). Esses valores foram calculados pelos coeficientes de digestibilidade estimados de $96 \%$ para EE e ENN e de $91 \%$ para proteínas (HARRIS, 1966).

O coeficiente de digestibilidade (CD) é a proporção de nutriente consumido que, na realidade, está disponível para a absorção e utilização pelo organismo do animal (CASE; CAREY; HIRAKAWA, 1998).

Kendall, Burger e Smith (1985) coletaram dados de digestibilidade em cães de 106 amostras de alimentos comerciais secos, semi-úmidos ou enlatados e observaram que os coeficientes médios de digestibilidade das proteínas, do extrato etéreo (medição do conteúdo lipídico) e do extrato nãonitrogenado (medição do conteúdo de carboidratos solúveis) foram de 81,85 e $79 \%$, respectivamente.

A utilização dos fatores de Atwater para determinar o conteúdo de EM para cães e gatos tende a superestimar as cifras da EM de vários alimentos. Este cálculo equivocado ocorre porque a digestibilidade de muitos ingredientes para animais domésticos é inferior à digestibilidade da maioria dos alimentos consumidos por humanos, fazendo com que os fatores de Atwater sejam imprecisos para a utilização no cálculo da EM dos alimentos para animais (CASE; CAREY; HIRAKAWA, 1998). 
O NRC (1985) recomenda o emprego de coeficientes de digestibilidade de 80, 90 e 85\% para proteínas, lipídios e carboidratos nos alimentos preparados para cães.

Quando se reajustaram os valores da EB em função da digestibilidade e das perdas urinárias, designaram-se valores da EM de 3,5; 8,5 e 3,5 kcal/g para proteínas, lipídios e carboidratos, nessa ordem (TABELA 1) (CASE; CAREY; HIRAKAWA, 1998; NRC, 1985).

TABELA 1 - Fatores e coeficientes de digestibilidade (CD) utilizados na espécie canina para carboidratos, proteínas e lipídios.

Table 1 - Digestibility factors and parameters used in dogs for carbohydrates, proteins and lipids

\begin{tabular}{l|c|c|c|c}
\hline Nutriente & $\begin{array}{c}\text { CD dos } \\
\text { alimentos para } \\
\text { humanos }\end{array}$ & Fator de Afwater & $\begin{array}{c}\text { CD dos alimentos } \\
\text { para animais }\end{array}$ & $\begin{array}{c}\text { Fator de Afuvaler } \\
\text { modificado }\end{array}$ \\
\hline Carboidratos & $96 \%$ & $4 \mathrm{kcal} / \mathrm{g}$ & $85 \%$ & $3,5 \mathrm{kcal} / \mathrm{g}$ \\
Proteinas & $91 \%$ & $4 \mathrm{kcal} / \mathrm{g}$ & $80 \%$ & $3,5 \mathrm{kcal} / \mathrm{g}$ \\
Lipidios & $96 \%$ & $9 \mathrm{kcal} / \mathrm{g}$ & $90 \%$ & $8,5 \mathrm{kcal} / \mathrm{g}$ \\
\hline
\end{tabular}

Fonte: CASE; CAREY; HIRAKAWA, 1998.

Ainda que estes facilitem uma estimativa mais adequada dos valores da EM dos alimentos para cães do que a calculada mediante os fatores de Atwater, ainda é possível que subestimem os valores da EM dos alimentos de alta qualidade, que contenham proteínas muito digeríveis e baixos níveis de fibras nãodigeríveis. Ao contrário, o valor da EM dos alimentos que contêm quantidades elevadas de fibra vegetal e/ou carne de baixa qualidade será ligeiramente superestimada por estes fatores (NRC, 1985).

Muitos trabalhos tentaram definir o melhor método (fórmula) para se estimar a energia metabolizável dos alimentos para cães, podendo, deste modo, facilitar a determinação de quantidades diárias a serem fornecidas aos cães, independente do estágio fisiológico ou nível de atividade deste animal.

\section{Determinação da energia metabolizável}

A determinação de uma equação exata para calcular as necessidades energéticas dos cães é uma tarefa muito complexa devido à grande variedade de tamanho e peso corporal observada na espécie canina.

Segundo Case, Carey e Hirakawa (1998), a quantidade de energia utilizada pelo organismo está relacionada com a superfície corporal total. A superfície corporal por unidade de peso aumenta com o aumento no tamanho do animal. Como conseqüência, o requerimento energético de animais com pesos corporais muito diferentes não se relaciona corretamente com os valores de peso vivo; na verdade, relaciona-se com o peso corporal elevado a certa potência específica, denominado peso metabólico. O peso metabólico visa a nivelar as diferenças de superfície corporal dos animais de tamanhos muito diferentes.

Autores como Kendall, Burger e Smith (1985), Kienkle et al. (1998), Kienkle e Rainbird (1991), Mânner (1991), Earle (1993), Heusner (1987) e Hill (1993), por meio de equações de regressão baseadas no peso corporal, estimaram a potência mais adequada para determinar a EM de alimentos para cães nas mais diferentes fases da vida e níveis de atividade.

O NRC (1985) e Case, Carey e Hirakawa (1998), baseados nas equações determinadas por aqueles autores, observaram que a função de potência 0,67 é a mais adequada. Dessa forma, chegaram à equação alométrica descrita a seguir:

$$
\text { Necessidade de } \mathrm{EM}=\mathrm{K} \times \mathrm{W}_{\mathrm{kg}}^{0,67} \mathrm{kcal} / \mathrm{dia}
$$

onde,

$\rightarrow \mathrm{K}=$ fator de correção para diferentes níveis de atividade

$132 \rightarrow$ animal inativo (sedentário); 
$145 \rightarrow$ animal moderadamente ativo;

$200 \rightarrow$ animal muito ativo;

$300 \rightarrow$ rendimento para resistência (cães de corrida).

$\rightarrow \mathrm{W}=$ peso vivo do animal.

Esta equação proporciona uma estimativa precisa dos requerimentos energéticos diários para diferentes tamanhos de cães adultos em diferentes níveis de atividade.

Já a equação descrita pelo NRC (1974), que utiliza a função de potência 0,75 determinada por Kleiber (1947), proporciona boa estimativa para raças de tamanho pequeno e médio, durante a fase de manutenção. A equação descrita é a seguinte $\mathrm{EM}=132 \times \mathrm{PV}^{0,75}$.

Outros autores também definiram equações para a predição da EM. Burger (1994) trabalhou com 48 cães da raça Border Collies, de trabalho e companhia, no Waltham Centre for Pet Nutrition (WCPN $U K)$. Observou a exigência energética diária (EED) destes animais (Tabela 2) e concluiu que a exigência energética média diária, quando considerados todos os animais, ficou muito próxima da fórmula EED = $125,1 \mathrm{PV}^{0,75}$ (kcal/dia), preconizada pelo $W C P N$ para cães adultos em atividade moderada.

TABELA 2 - Requerimentos energéticos de cães do Waltham Centre for Pet Nutrition (WCPN) - Experimento com Border Collies

Table 2 - Dogs energetic requirements from Waltham Centre for Pet Nutrition (WCPN) Experiment with Border Collies

\begin{tabular}{l|l|r}
\hline Cães & Exercicio & EED $^{*}(\mathrm{kcal} / \mathrm{dia})^{1}$ \\
\hline 9 & Baixo $(<1 \mathrm{~h})$ & $97,1 \mathrm{PV}^{0,75} \pm 41,1$ \\
28 & Moderado $(1-3 \mathrm{~h})$ & $124,2 \mathrm{PV}^{0,75} \pm 44,5$ \\
10 & Alto $(3-6 \mathrm{~h})$ & $175,1 \mathrm{PV}^{0,75} \pm 85,4$ \\
47 & Média & $130 \mathrm{PV}^{0,75} \pm 60,3$ \\
\hline
\end{tabular}

Fonte: Adaptado de BURGER, 1994.

* EED - Exigência Energética Diária.

${ }^{1}$ valores médios $\pm \mathrm{DP}$.

A diversidade entre cada indivíduo e as condições ambientais sob as quais cada cão é mantido pode originar necessidades até $25 \%$ superiores ou inferiores aos valores estimados pelas equações indicadas anteriormente (CASE; CAREY; HIRAKAWA, 1998).

\section{Consumo energético}

Todos os animais necessitam de fonte constante de energia no alimento para sobreviver. As plantas absorvem a radiação solar, transformando-a em nutrientes que contêm energia. Alguns animais consomem plantas e utilizam diretamente a sua energia, ou então transformam os seus nutrientes em outras moléculas energéticas. As plantas armazenam energia principalmente na forma de carboidratos; os animais, por sua vez, utilizam os lipídios como principal depósito de energia (ANDRIGUETTO et al., 1983).

Segundo Case, Carey e Hirakawa (1998), para que o trabalho metabólico do organismo ocorra, é necessário que haja uma fonte de energia. Neste trabalho metabólico estão incluídas a manutenção e síntese dos tecidos orgânicos, a atividade física e a termorregulação.

Devido à sua grande importância, fica fácil entender que a demanda por energia seja sempre a primeira a ser satisfeita pelo alimento de um animal. Independentemente das necessidades específicas que os cães tenham de ácidos graxos provenientes dos lipídios do alimento ou de aminoácidos essenciais procedentes das proteínas do alimento, os componentes energéticos do alimento serão utilizados, primeiramente, para satisfazer as demandas energéticas.

Requerimento energético é a ingestão de energia necessária para suprir o gasto energético normal. Uma vez satisfeitas essas demandas, os nutrientes restantes serão empregados em outras funções metabólicas (CASE; CAREY; HIRAKAWA, 1998; MILLER et al., 1965). 
Keyes (1950) observou três requerimentos: (a) requerimento do animal no seu estado atual; (b) requerimento teórico do animal para manter seu tamanho corporal e nível de atividade ideal e (c) requerimento para corrigir alterações no tamanho e peso corporal ou falhas no metabolismo decorrentes de uma doença.

Sabendo-se da influência da energia sobre o consumo de alimentos, deve-se entender que diversos fatores influem sobre o consumo energético total diário de um animal (QUADRO 3).

\begin{tabular}{l|l}
\hline Classe & Mamiferos \\
\hline Subclasse & Eutérios \\
Superordem & Camivoros \\
Ordem & Camivoros terrestres \\
Familia & Canideos \\
Gênero & Canis \\
Espécie & Canis familiaris (2n $\rightarrow 78$ cromossomos) \\
\hline
\end{tabular}

QUADRO 3 - Fatores que afetam os componentes do consumo energético animal Chart 3 - Factors that affect the animal energetic consumption components

Fonte: CASE; CAREY; HIRAKAWA, 1998.

TMR - quantidade de energia consumida enquanto o animal permanece sentado com tranqüilidade em um ambiente confortável, várias horas depois de uma refeição ou de uma atividade física;

AMV - trabalho físico realizado em uma determinada carga por um determinado período;

TIC - calor produzido após a ingestão de um alimento;

TA - consumo energético adicional para adaptar o organismo do animal a alterações ambientais.

\section{CONCLUSÕES}

A determinação da exigência energética para cães adultos não é uma tarefa fácil. A espécie canina apresenta grande variação de tamanho entre os diferentes representantes de sua espécie. Podem ser encontradas raças com $1 \mathrm{~kg}$, como é o caso do Pinscher, até cães com mais de $80 \mathrm{~kg}$ de peso vivo, como no caso dos cães da raça São Bernardo.

Com base nestas informações e características inerentes à espécie canina, pode-se chegar às seguintes conclusões:

a. Existem diversas equações para predição da necessidade energética diária ou energia de um alimento para cães.

b. Destas equações, uma que pode ser utilizada como ponto de partida é a equação alométrica descrita por Case et al. (1998) - Necessidade de $\mathbf{E M}=\mathbf{K} \mathbf{x} \mathbf{W}_{\mathrm{kg}}^{0,67} \mathrm{kcal} / \mathrm{dia}$ onde:

$\mathrm{K}=$ fator de correção para diferentes níveis de atividade

132 = animal inativo (sedentário);

145 = animal moderadamente ativo;

200 a animal muito ativo;

$300=$ rendimento para resistência (cães de corrida).

$\mathrm{W}=$ peso vivo do animal.

c. A determinação desta demanda de energia por meio de equações e fórmulas matemáticas serve basicamente como um ótimo ponto de partida para a implantação de um programa alimentar de curto, médio ou longo prazo.

d. A utilização deste expediente não elimina a necessidade de se realizar um acompanhamento - por um profissional especializado - do desempenho e comportamento do animal em questão. Só assim, por meio da utilização do máximo de informações disponíveis, é que se pode garantir a saúde e longevidade dos cães com o máximo de qualidade de vida. 


\section{REFERÊNCIAS}

AAFCO. Official Publication of the Association of American Feed Control Officials Incorporated. Atlanta, 1999. 162 p.

AHLSTROM, O.; SKREDE, A. Comparative nutrient digestibility in dogs, blue foxes, mink and rats. Journal of Nutrition, v. 128, p. 2676-2677, 1998.

ANDRIGUETTO, J. M. et al. Cães. In: p. 353-363. cap. 8, v. 1.

. Nutrição animal. 5. ed. São Paulo: Nobel, 1983.

BURGER, I. H. Energy needs of companion animals: matching food intakes to requirements throughout the life cycle. Journal of Nutrition, v. 124, p. 2584-2593, 1994.

BURGER, I. H.; JOHNSON, J. V. Dogs large and small: The allometry of energy requirements within a single species. Journal of Nutrition, v. 121, p. 18-21, 1991.

CASE, L. P.; CAREY, D. P.; HIRAKAWA, D. A. Nutrição canina e felina. Madrid: Harcourt Brace de España S. A., 1998, 424 p.

CONSTABLE, P. D. et al. Factors associated with finishing status for dogs competing in a long-distance sled race. Journal of American Veterinary Medicine Association, v. 208, n. 6, p. 879-882, 1996.

EARLE, K. E. Calculations of energy requirements of dogs, cats and small psittacine birds. Journal of Small Animal Practice, Leicestershire, v. 34, p. 163-173, 1993.

GRANDJEAN, D. Enciclopédia do cão - Royal Canin. Paris: Aniwa S. A., 2001. 655 p.

HARRIS, L. E. Biological energy interrelationships and glossary of energy terms. Washington, D. C.: National Academy of Sciences and National Academy Press, 1966. 85 p.

HEUSNER, A. A. What does the power function reveal about structure and function in animals of different size? Annual Review of Physiology, v. 49, p. 121-133, 1987.

HILL, R. C. A rapid method of estimating maintenance energy requirement from body surface area in inactive adult dogs and in cats. Journal of American Veterinary Medicine Association, v. 202, n.11, p.1814-1816, 1993.

KENDALL, P. T. Comparative evaluation of apparent digestibility in dogs and cats. Procedings of Nutritional Society, v. 40, p. 45, 1981.

KENDALL, P. T.; BURGER, I. H.; SMITH, P. M. Methods of estimation of the metabolizable energy content of cat food. Feline Practice, v. 15, p. 38-44, 1985.

KENDALL, P. T.; HOLME, D. W.; SMITH, P. M. Comparative evaluation of net digestive and absorptive efficiency in dogs and cats fed a variety of contrasting diet types. Journal of Small Animal Practice, v. 23, p. 577-587, 1982.

KEYES, A. Energy requirements in human adults. Journal of American Veterinary Medicine Association, v. 122, p. 333, 1950.

KIENKLE, E. et al. An improved method for the estimation of energy in pet foods. Journal of Nutrition., v. 128, p. 2806-2808, 1998.

KIENKLE, E.; RAINBIRD, A. Maintenance energy requirement of dogs: what is the correct value for the calculation of metabolic body weight in dogs? Journal of Nutrition, Germany, v. 121, p. 39-40, 1991.

MÂNNER, K. Energy requirement for maintenance of adult dogs. Journal of Nutrition, v. 121, p. 3738, 1991. 
MCDONALD, P.; EDWARDS, R. A.; GREENHALGH, J. F. D. Animal nutrition. $4^{\text {th }}$ ed. Harlow: Longman Scientific and Technical, 1988. 320 p.

MILLER, H. Essential nutrients and their role in metabolism. Basic guide to canine nutrition. New York: Gaines Dog Research Center, 1965. cap. 3, p. 12-14.

MOHRMAN, R. K. Alimentação de cães: nutrição e criação de cães e gatos. São Paulo: Purina Alimentos, 1979. cap. 2.

NRC. Nutrient requirements of dogs. Washington: National Academy of Sciences, 1974. 83 p.

NRC. Nutrient requirements of dogs. Washington: National Academy Press, 1985. 79 p.

NRC. Nutritional energetic of domestic animals and glossary of energy terms. Washington: National Academy Press, 1981. 80 p.

Recebido em: 30/05/2007

Received in: 05/30/2007

Aprovado em: 31/07/2007

Approved in: 07/31/2007 